\title{
A rare case of Candida parapsilosis osteomyelitis: a literature review and proposed treatment algorithm
}

\author{
John Michael Yingling, Li Sun, Richard Yoon ${ }^{*}$ and Frank Liporace
}

\begin{abstract}
Background: Candida parapsilosis is a rare opportunistic pathogen that can be found in immunosuppressed patients. There are seldom-reported cases of fungal osteomyelitis surrounding orthopedic implants.

Case presentation: This is a case of chronic Candida parapsilosis osteomyelitis in an immunocompromised patient with a prior open reduction and internal fixation for a closed bimalleolar ankle fracture that went on to neglected wound complications. The patient underwent series of treatments including removal of hardware, serial irrigation and debridements, negative pressure wound therapy, and intravenous antifungal therapy. Our case illustrates the possibility of this rare pathogen involved in orthopedic surgery particularly in immunocompromised hosts.

Conclusion: Fungal and atypical pathogens should always be considered in such patients or if another diagnosis is not clear. Protracted time to culture specimens should be considered for at least four weeks in such situations. This article outlines a review of the literature and treatment algorithm to guide physicians when managing patients with this rare infection.
\end{abstract}

Keywords: Acute and chronic deep fungal infection management, Exposed hardware, Fungus, Irrigation and debridement, Negative pressure wound therapy, Removal of hardware, Saucerization, Ankle fracture

\section{Background}

Staphylococcus aureus is the most common infecting organism found in adults with osteomyelitis [1]. Salmonella osteomyelitis has long been associated with SS or $\mathrm{SC}$ hemoglobinopathies [2]. Fungal osteomyelitis is seen increasingly in chronically ill patients receiving longterm intravenous therapy or parenteral nutrition and the immunocompromised [3]. The most common fungal osteomyelitis pathogens are Candida species. $C$ albicans is the most common species isolated in culture $[4,5]$. Healthy subjects can harbor fungal pathogens on their skin, in their GI tract, sputum, or other body fluids, and can present with musculoskeletal fungal infections, but most only become pathogenic in a host with a depressed immune system $[4,6]$. Most resources will agree Human Immunodeficiency Virus (HIV) infection, cirrhosis, cancer, diabetes, prolonged corticosteroid use, alcoholism,

\footnotetext{
* Correspondence: yoonrich@gmail.com
Division of Orthopaedic Trauma \& Adult Reconstruction, Department of
Orthopaedic Surgery, Jersey City Medical Center - RWJBarnabas Health,

* Correspondence: yoonrich@gmail.com
Division of Orthopaedic Trauma \& Adult Reconstruction, Department of
Orthopaedic Surgery, Jersey City Medical Center - RWJBarnabas Health,

* Correspondence: yoonrich@gmail.com
Division of Orthopaedic Trauma \& Adult Reconstruction, Department of
Orthopaedic Surgery, Jersey City Medical Center - RWJBarnabas Health, Jersey City, NJ 07302, USA
}

(c) The Author(s). 2017 Open Access This article is distributed under the terms of the Creative Commons Attribution 4.0 International License (http://creativecommons.org/licenses/by/4.0/), which permits unrestricted use, distribution, and reproduction in any medium, provided you give appropriate credit to the original author(s) and the source, provide a link to the Creative Commons license, and indicate if changes were made. The Creative Commons Public Domain Dedication waiver (http://creativecommons.org/publicdomain/zero/1.0/) applies to the data made available in this article, unless otherwise stated. and renal failure are among predisposing factors for funof candida osteomyelitis, a majority of the patients were not heavily immunosuppressed (neutropenic, transplant patients, underlying hematologic malignancy etc.) [4] Risk factors for invasive candidiasis include recent surgery, intravenous drug abuse (IVDA), broad spectrum antibiotic use, chemotherapy, parenteral nutrition, and central venous catheters [3]. Candida osteomyelitis develops from hematogenous spread of infection in approximately $70 \%$ of cases and can present up to 3 years after bloodstream infection [5]. Direct inoculation occurs in about $24 \%$, and contiguous infection in less than $10 \%$ of cases. The diagnosis can often be difficult. Traditional laboratory inflammatory markers such as white blood cell count (WBC), erythrocyte sedimentation rate (ESR), and C-reactive protein (CRP) levels could only be slightly elevated or even normal. Clinically, the majority of patients will present with pain, tenderness, erythema, and decreased functionality of the extremity, but rarely with fever or draining wound. Candida osteomyelitis is 
diagnosed by culture via direct biopsy in over $70 \%$ of cases [4]. Interestingly, in patients with disseminated candidiasis, only $30 \%$ to $50 \%$ have a positive blood culture, which shows the difficulty in isolating the organism by blood culture. [7] Fungal infections are often complicated by concomitant bacterial infections, Staphylococcus aureus being the most common, but also included is Proteus mirabolis [4]. Fluconazole has been used to successfully treat candida osteomyelitis in spine patients and is considered to have a good safety and tolerability profile. Voriconazole may be used for fluconazole resistant infections and is said to have good bone penetration $[3,8,9]$. Adding to the difficulty of treating Candida infections, some species, namely albicans and parapsilosis, share the ability to form biofilms that may be resistant to standard -azole antifungals. Importantly, Echinocandin agents like Caspofungin or Micafungin have a unique mechanism of action that allows them to be used in combination with other antifungals and may be effective against biofilms namely involved with retained hardware $[3,10-12]$. Traditionally surgical intervention has involved debridement of all necrotic tissue including bone and sinus tracts with specimens sent for culture and pathology, irrigation with copious amounts of sterile fluid, and placement of antifungal and/or antibiotic beads if necessary. Based on the stability of the bone or ability to stabilize with external methods, such as splinting, all internal hardware may be removed [3, 4, 13]. $90 \%$ of patients have complete or at least partial response to treatment with surgery and antifungal therapy [4]. This is a report of a patient's presentation, course, and treatment that will contribute to the paucity of literature on Candida parapsilosis Osteomyelitis.

\section{Case presentation}

An adult African American female was admitted to our institution for altered mental status and hypotension in October of 2015. Exposed hardware on her left ankle was noted from a previous open reduction and internal fixation 21 years prior.

The patient lived in a nursing home and her past medical history included end stage renal disease requiring dialysis three times per week, HIV on Highly Active Antiretroviral Therapy (HAART) diagnosed 10 years earlier, Hepatitis B and $C$, hypertension, coronary artery disease status post internal defibrillator placement, congestive heart failure, history of cerebral vascular accidents, anemia of chronic disease, seizure disorder, and dementia. The patient was noted to have exposed hardware of her left ankle on visits as far back as 9 years prior to the discussed admission to our institution.

During her initial work up the diagnosis of multilobular pneumonia was made and she was started on linezolid and aztreonam while initial blood cultures were negative. The

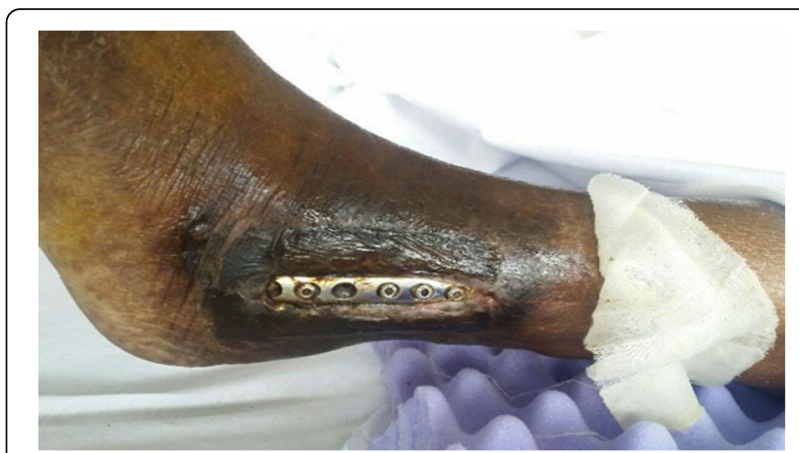

Fig. 1 Left ankle displaying exposed hardware with no drainage or erythema

patient went on to respiratory failure and septic shock secondary to her multilobular pneumonia, was subsequently intubated, and treated with vasopressors and antibiotics. The following day her sputum cultures grew Candida albicans and fluconazole was added to her treatment. Eight days later the patient showed improvement and was extubated still requiring vasopressors and hemodialysis. Clindamycin was added to the antibiotic regiment. Six days later Orthopedics was consulted to evaluate the exposed hardware of the lateral incision site of her left ankle (Fig. 1). The wound was dry, showed no signs of acute infection (erythema, purulent drainage, fluctuance, or warmth). The Radiographs showed retained medial and lateral hardware (Fig. 2). The following day the patient was taken to the operating room for removal of the exposed fibular plate and screws and a thorough irrigation and debridement of necrotic tissue. Intra-operative samples of peri-implant tissues and bone were sent for gram stain and culture. A negative pressure wound therapy dressing (NPWT) (V.A.C. Ulta Negative Pressure Wound Therapy System, KCI San Antonio, TX) was placed sterilely in the operating room. The NPWT dressing was changed at bedside every two days. The intraoperative bone sample cultures grew Candida parapsilosis and the soft tissue cultures grew Candida parapsilosis and Proteus Mirabilis. The NPWT dressing was then discontinued and the wound was dressed with sterile $4 \times 4$ gauze soaked with $0.25 \%$ Dakin's solution. Under the direction of our infectious disease specialist the

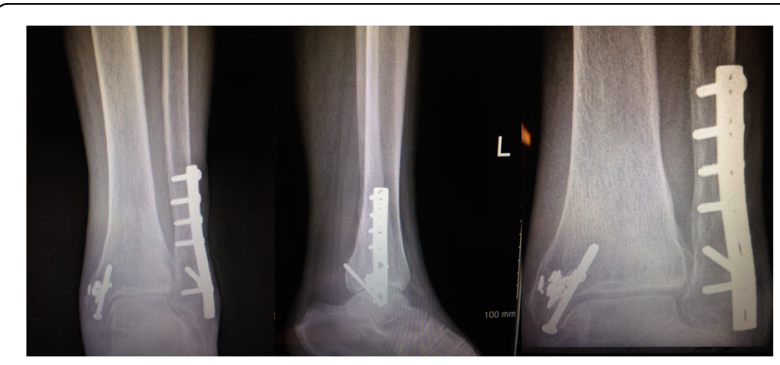

Fig. 2 Preoperative AP, lateral, and mortise view of left ankle demonstrating retained hardware 


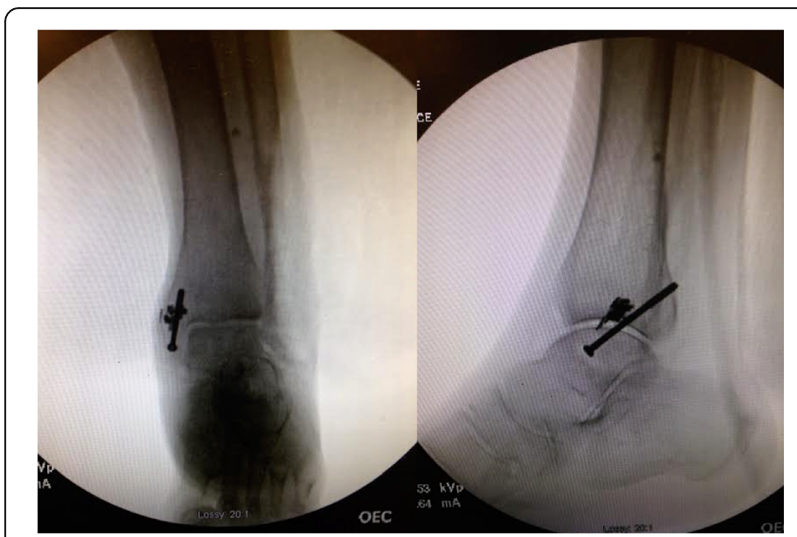

Fig. 3 Intra-operative fluoroscopic images demonstrating the removal of fibular hardware and saucerization of the bone

fluconazole was continued for coverage of both candida species. A week later the patient was taken to the operating room for another irrigation and radical debridement of necrotic bone, saucerization, and wound closure. Intraoperative fluoroscopic images are shown in (Fig. 3). A NPWT dressing was placed with scheduled changes on floor every three days. A variable course treating the patient's sepsis and multiple medical issues complicated by bilateral upper extremity deep vein thrombosis ensued. The patient was intubated and transferred to the Intensive Care Unit on multiple vasopressors for closer monitoring after pulseless apneic episodes. At this time Caspofungin was added to her antifungal regiment of fluconazole along with other stronger broad-spectrum antibiotics. The patient eventually expired from her medical comorbidities.

\section{Discussion}

Candida osteomyelitis has been described in the literature primarily as Candida albicans species. This rare case of Candida parapsilosis osteomyelitis in an immunosuppressed patient is one of the few reported cases to be found. It is important to bring this to clinicians' attention in order to keep it as differential diagnosis when treating similar patients in the future. Fungal osteomyelitis is difficult to diagnose in any patient with retained orthopedic hardware. Patients may often present with an equivocal clinical picture, normocytic labs, normal vital signs, unchanged radiographs, and wounds with minimal drainage. During the initial work up of a patient with negative blood cultures and risk factors for fungal infections you must

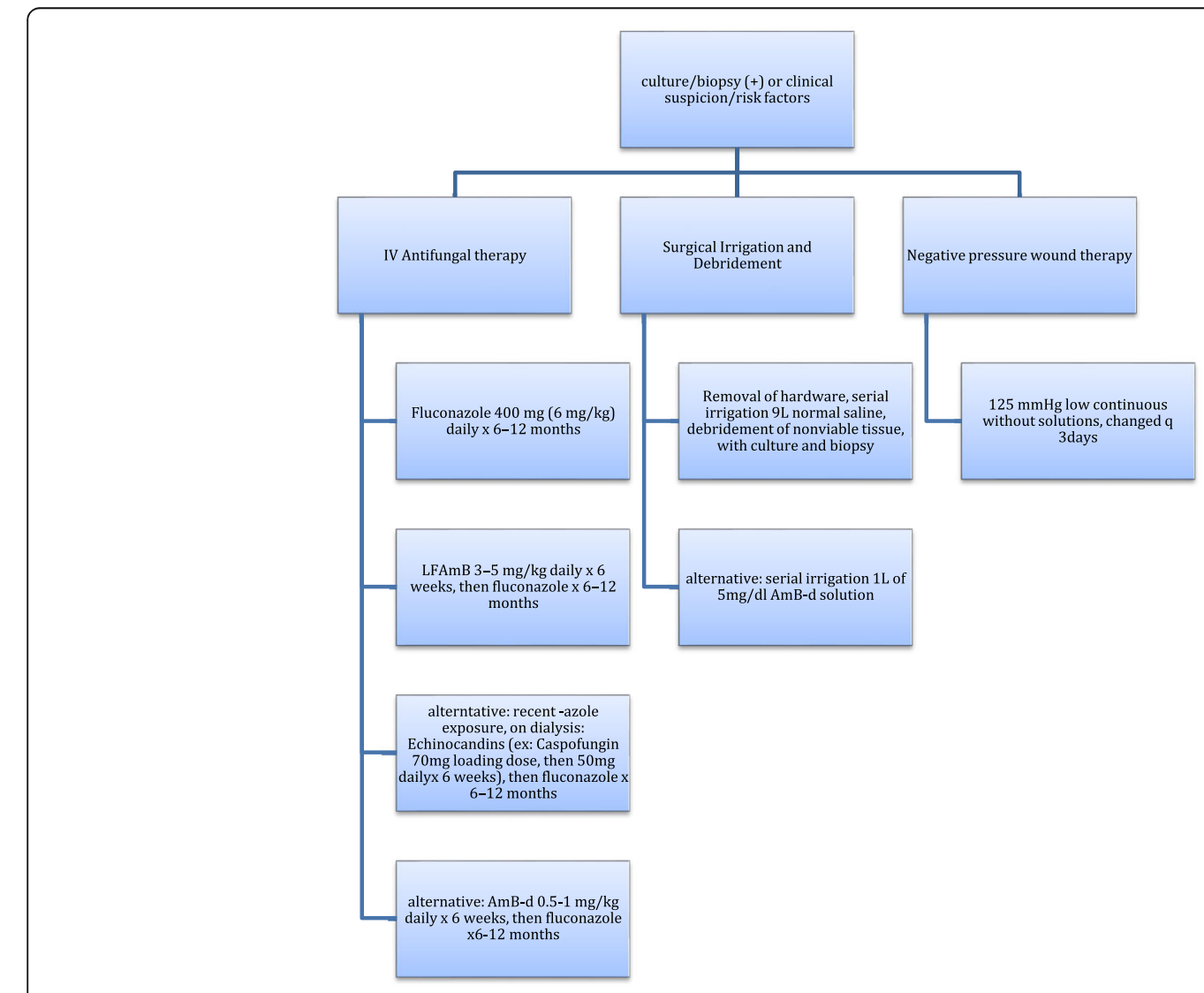

Fig. 4 Algorithm for treating peri-implant Candida parapsilosis osteomyelitis 
maintain a high clinical suspicion for fungal osteomyelitis. It is important to keep fungal cultures for up to 4 weeks, as they may not initially demonstrate a pathogen [14]. The current recommendations of the Infectious Disease Society of America (IDSA) recommend 6-12 months of systemic antifungal agents in conjuncture with surgical debridement usually in the form of Fluconazole $400 \mathrm{mg}$ (6 mg/kg) daily for 6-12 months. Lipid formulation of Amphotericin B 3-5 mg/kg daily for several weeks, then fluconazole for 6-12 months is also recommended, or an Echinocandin or Amphotericin B-d 0.5-1 mg/kg daily for several weeks then fluconazole for 6-12 months as an alternative, with the European recommendations being similar [12, 15-17]. Echinocandins have only parenteral routes of administration but have a very safe profile and do not need dosing for renal impairment or dialysis as they are eliminated through non-enzymatic degradation [17]. If financial or time constrains arise it may be reasonable to administer a shorter duration of IV antifungal therapy (AFT) as long as you ensure a thorough surgical debridement. Miller et al. described a mean duration of 45 days of IV AFT with surgical debridement in twentythree cases and reported 90\% good outcomes with no relapsing infections. Concomitant bacterial infection may convolute the picture but should be treated with culture sensitivity and specificity guided IV antibiotics along with the AFT. Due to the ability of Candida species to form biofilms it is important to remove any retained implants if the stability of the bone is adequate or maintained by other means such as splinting. Some have proposed using $1 \mathrm{~L}$ of $5 \mathrm{mg} / \mathrm{dl}$ Amphotericin B Deoxycholate irrigation solution with continuous suction without subsequent saline washes. They reported no complications or systemic toxicity but also have no comparisons in their study [16]. In the interim we used $0.25 \%$ Dakin solution, or Sodium Hypochlorite, as a topical antiseptic adjunct. Dakin solution, though controversially may have negative effects on neutrophil chemotaxis and fibroblast viability, is bactericidal and can be useful in the setting of an immunocompromised patient [18-20]. In order to enhance the penetration of IV AFT and blood flow to the infected area, a thorough surgical removal of all unviable bone and soft tissue from the area is recommended. This usually will require serial debridement at time intervals often dictated by the patient's ability to tolerate surgery. During the initial and all subsequent procedures it is important to obtain culture samples along with tissue biopsy for histologic evaluation and monitoring for eradication of the pathogen as blood culture, laboratory markers, and clinical signs may be inconsistent and unreliable often due to the patient's inability do respond. Based on current literature and the personal experience at our institution we propose the following algorithm for treating peri-implant Candida parapsilosis osteomyelitis in the Fig. 4 provided.

\section{Conclusion}

Fungal and atypical pathogens should always be considered in such patients or if another diagnosis is not clear. Protracted time to culture specimens should be considered for at least four weeks in such situations. Treatment should consist of early antifungal medication, serial irrigation and debridement, and negative pressure wound therapy using the proposed algorithm.

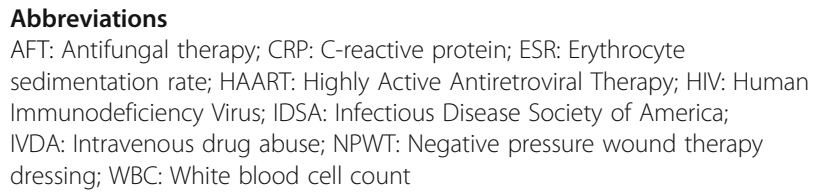

Funding

The authors declare no funding was provided for current manuscript.

Availability of data and materials

The datasets used and/or analyzed during the current study are available from the corresponding author on reasonable request.

\section{Authors' contributions}

The authors FL and RY were responsible for study design, FL was primary operating surgeon and responsible for all clinical decisions. JY and LS were responsible for coordination and drafting of the manuscript. All authors read and approved the final manuscript.

\section{Ethics approval and consent to participate}

All procedures and interventions were performed under informed consent.

\section{Consent for publication}

Written informed consent was obtained by the patient and described in the article and is available to the Editor-in-Chief by request.

\section{Competing interests}

The authors declare that they have no competing interests.

\section{Publisher's Note}

Springer Nature remains neutral with regard to jurisdictional claims in published maps and institutional affiliations.

Received: 30 September 2017 Accepted: 5 December 2017

Published online: 21 December 2017

\section{References}

1. Cierny G 3rd, Mader JT, Penninck JJ. A clinical staging system for adult osteomyelitis. Clin Orthop Relat Res. 2003;414:7-24.

2. McAnearney S, McCall D. Salmonella Osteomyelitis. Ulster Med J. 2015;84(3):171-2.

3. Bariteau JT, Waryasz GR, McDonnell M, Fischer SA, Hayda RA, Born CT. Fungal osteomyelitis and septic arthritis. J Am Acad Orthop Surg. 2014;22(6): 390-401.

4. Gamaletsou MN, Kontoyiannis DP, Sipsas NV, Moriyama B, Alexander E, Roilides E, et al. Candida osteomyelitis: analysis of 207 pediatric and adult cases (1970-2011). Clin Infect Dis. 2012;55(10):1338-51.

5. Slenker AK, Keith SW, Horn DL. Two hundred and eleven cases of Candida osteomyelitis: 17 case reports and a review of the literature. Diagn Microbiol Infect Dis. 2012;73(1):89-93.

6. Cuellar ML, Silveira LH, Espinoza LR. Fungal arthritis. Ann Rheum Dis. 1992; 51(5):690-7.

7. Kohli R, Hadley S. Fungal arthritis and osteomyelitis. Infect Dis Clin N Am. 2005;19(4):831-51.

8. Cho K, Lee SH, Kim ES, Eoh W. Candida Parapsilosis spondylodiscitis after lumbar discectomy. J Korean Neurosurg Soc. 2010;47(4):295-7.

9. Katragkou A, Chatzimoschou A, Simitsopoulou M, Dalakiouridou M, DizaMataftsi E, Tsantali C, et al. Differential activities of newer antifungal agents 
against Candida Albicans and Candida Parapsilosis biofilms. Antimicrob Agents Chemother. 2008;52(1):357-60.

10. Baillie GS, Douglas $L$. Candida biofilms and their susceptibility to antifungal agents. Methods Enzymol. 1999:310:644-56.

11. Kuhn DM, George T, Chandra J, Mukherjee PK, Ghannoum MA. Antifungal susceptibility of Candida biofilms: unique efficacy of amphotericin B lipid formulations and echinocandins. Antimicrob Agents Chemother. 2002;46(6): 1773-80.

12. Ramage G, Vande Walle K, Wickes BL, Lopez-Ribot JL. Standardized method for in vitro antifungal susceptibility testing of Candida Albicans biofilms. Antimicrob Agents Chemother. 2001:45(9):2475-9.

13. Legout L, Assal M, Rohner P, Lew D, Bernard L, Hoffmeyer P. Successful treatment of Candida Parapsilosis (fluconazole-resistant) osteomyelitis with caspofungin in a HIV patient. Scand J Infect Dis. 2006;38(8):728-30.

14. Bosshard PP. Incubation of fungal cultures: how long is long enough? Mycoses. 2011;54(5):e539-45.

15. Cornely OA, Bassetti M, Calandra T, Garbino J, Kullberg BJ, Lortholary O, et al. ESCMID* guideline for the diagnosis and management of Candida diseases 2012: non-neutropenic adult patients. Clin Microbiol Infect. 2012; 18(Suppl 7):19-37.

16. Miller AO, Gamaletsou MN, Henry MW, Al-Hafez L, Hussain K, Sipsas NV, et al. Successful treatment of Candida osteoarticular infections with limited duration of antifungal therapy and orthopedic surgical intervention. Infect Dis (Lond). 2015:47(3):144-9.

17. Pappas PG, Kauffman CA, Andes D, Benjamin DK Jr, Calandra TF, Edwards JE $\mathrm{Jr}$, et al. Clinical practice guidelines for the management of candidiasis: 2009 update by the Infectious Diseases Society of America. Clin Infect Dis. 2009; 48(5):503-35.

18. Cooper ML, Laxer JA, Hansbrough JF. The cytotoxic effects of commonly used topical antimicrobial agents on human fibroblasts and keratinocytes. J Trauma. 1991;31(6):775-82. discussion 82-4

19. Doughty D. A rational approach to the use of topical antiseptics. J Wound Ostomy Continence Nurs. 1994;21(6):224-31.

20. McCullough M, Carlson GW. Dakin's solution: historical perspective and current practice. Ann Plast Surg. 2014;73(3):254-6.

\section{Submit your next manuscript to BioMed Central and we will help you at every step:}

- We accept pre-submission inquiries

- Our selector tool helps you to find the most relevant journal

- We provide round the clock customer support

- Convenient online submission

- Thorough peer review

- Inclusion in PubMed and all major indexing services

- Maximum visibility for your research

Submit your manuscript at www.biomedcentral.com/submit
Biomed Central 